\title{
RÉGIMEN JURÍDICO DEL POSGRADO EN CHILE: MÁS AUTONOMÍA QUE REGULACIÓN
}

José Julio León* 


\section{RESUMEN}

El artículo analiza el marco normativo del posgrado en Chile, a la luz de los principios constitucionales vigentes, así como desde la naturaleza de esta especie de programas. Se argumenta que el diseño legal es correcto, al establecer un predominio de la autonomía académica de las universidades por sobre las potestades regulatorias del Estado. Plantea también la urgencia de desarrollar la cúspide del sistema de educación superior, donde las necesidades de fiscalización son claramente menores que en la base (educación básica y media), puesto que los usuarios cuentan con mayor información e incentivos para elegir y discriminar el servicio que quieren contratar.

\section{ABSTRACT}

This is an analysis of the regulatory framework governing postgraduate studies in Chile covering the constitutional principles in force and also the nature of this type of programs. The paper holds that the legal plan has been adequately designed since it establishes the predominance of academic independence of universities over and above the regulatory powers of the state. It pays special attention to the need to further encourage the development of graduate programs in Chile and particularly the fact that this is the pinnacle of the higher education system where the need for monitoring is clearly less than at the base (elementary and secondary education) because students are more knowledgeable and have more incentives to chose and distinguish the educational service they wish to select. 


\section{RÉGIMEN JURÍDICO DEL POSGRADO EN CHILE: MÁS AUTONOMÍA QUE REGULACIÓN}

\section{ANTECEDENTES: DEFINICIONES Y DATOS}

Debemos comenzar estableciendo que el término posgrado alude convencionalmente a estudios sistemáticos que se llevan a cabo después de recibir el grado académico de licenciado, el que generalmente se vincula con la obtención del título profesional habilitante para el ejercicio de una determinada actividad. Sin embargo, dentro de la misma categoría existen diversos tipos de programas de estudio, adscritos también a distintas filosofías educacionales. Los expertos en la materia distinguen dos filosofías básicas: la que busca acrecentar el conocimiento y la que apunta a desarrollar aplicaciones concretas del conocimiento disponible. Los estudios de posgrado, a su turno, se clasifican en: doctorados, maestrías y especializaciones.

Los estudios conducentes al doctorado normalmente preparan a la persona para dedicarse a la actividad de investigación (acrecientan el conocimiento en un área del mismo). La especialización es un complemento de los estudios de licenciatura, que busca actualizar y profundizar los conocimientos del profesional en un área específica, con el fin de alcanzar una aplicación eficaz de los mismos al momento de resolver problemas prácticos. La maestría puede ser un primer paso al doctorado, o bien una combinación más o menos equilibrada de investigación y desarrollo profesional.

En Chile, como en general en Latinoamérica, predominan los estudios conducentes al grado de maestrías y las especializaciones. Ello, aun cuando tradicionalmente se ha justificado la creación de programas de posgrado en la necesidad de formar investigadores y cuadros académicos, lo que se logra a través del doctorado. 
Lo anterior induce a pensar que el desarrollo del nivel de posgrado ha respondido, en realidad, a necesidades diversas, tales como:

- Modernización del Estado y del sector productivo privado;

- Desarrollo económico y tecnológico;

- Mayor demanda por certificaciones, dado el carácter dinámico del empleo; y

- Búsqueda de prestigio y estatus, tanto institucional como personal.

El aumento en la demanda de profesionales especializados, por un lado, y la búsqueda de excelencia y prestigio institucional, por otro, llevó a las universidades chilenas a incrementar su oferta de posgrados, incluso sin contar con una sólida base de desarrollo en la respectiva área del conocimiento. Ello se advierte especialmente en la diversidad de programas de maestría y sus denominaciones, con referencia directa a las necesidades del mercado. El menor desarrollo de los doctorados, a su vez, se puede explicar por las siguientes razones:

- Autorregulación de universidades, al no cumplir con estándares internacionales para otorgar doctorados (se requiere que todos los académicos del programa tengan el grado de doctor y estén activos en investigación);

- Baja demanda, por costo, falta de becas y expectativas laborales;

- Requiere fuerte dedicación, por lo que es menos compatible con el trabajo profesional.

La matrícula total de posgrados ha aumentado considerablemente desde 1990, aunque todavía no podemos hablar de una masificación de él. Actualmente, las universidades tienen sobre 11.000 estudiantes, en programas de maestría y doctorado, de los cuales un treinta por ciento estudia en universidades privadas. Solo un bajo porcentaje estudia doctorados.

\section{EL CONTEXTO INSTITUCIONAL: AUTONOMÍA DE LAS UNIVERSIDADES Y LIBERTAD DE ENSEÑANZA}

De conformidad con la Constitución Política (CP), el Estado debe garantizar esferas de autonomía para que las personas $-y$ las organizaciones que éstas forman- puedan desarrollarse libremente y arbitrarse los medios para el logro de sus propios fines y la realización de sus proyectos o planes de vida. Así, nuestra Carta Fundamental establece en el artículo $1^{\circ}$ : 
a) "El Estado reconoce y ampara a los grupos intermedios a través de los cuales se organiza y estructura la sociedad y les garantiza la adecuada autonomía para cumplir sus propios fines específicos"; $y$

b) "El Estado está al servicio de la persona humana y su finalidad es promover el bien común, para lo cual debe contribuir a crear las condiciones sociales que permitan a todos y a cada uno de los integrantes de la comunidad nacional su mayor realización material y espiritual posible, con pleno respeto a los derechos y garantías que esta constitución establece".

En consecuencia, la CP garantiza la libertad de enseñanza, derecho que asiste a toda persona para participar en los procesos de enseñanza y aprendizaje, sea impartiendo o recepcionando conocimientos, sin otras limitaciones que las que imponen la moral, las buenas costumbres, el orden público y la seguridad nacional. La libertad de enseñanza comprende, entonces, el derecho a impartir conocimientos; el de elegir el contenido, sistema y métodos de la enseñanza; y la facultad de acreditar el grado de conocimientos adquiridos por los alumnos. Asimismo, ampara el bien jurídico de libertad de cátedra, entendido como la facultad del profesor para desarrollar las materias de un curso desde su personal enfoque, con prescindencia de criterios heterónomos.

La libertad de enseñanza, en cuanto incluye el derecho de abrir, organizar y mantener establecimientos educacionales, garantiza la autonomía académica, administrativa y económica de las instituciones de educación superior del Estado y de las particulares reconocidas por éste ${ }^{1}$. En tanto, la enseñanza con reconocimiento oficial está sujeta a algunas limitaciones adicionales a las que afectan a la enseñanza que no aspira a tal reconocimiento. Desde luego, según el inciso final de esta garantía, una ley orgánica constitucional establecerá los requisitos para el reconocimiento oficial de los establecimientos educacionales de todo nivel.

1 La referencia a la Autonomía Universitaria estaba incluida en el art. 10 No 17 de la Constitución de 1925, después de la Reforma de 1971 (Estatuto de Garantías Constitucionales); asimismo, figuraba en la propuesta de la Comisión de Estudios, pero fue sacada del texto definitivo de la Constitución de 1980. Lo anterior podría interpretarse como una intención positiva de disminuir el valor y el alcance de la autonomía; sin embargo, debe descartarse esta interpretación, en virtud de la protección general de la autonomía de los cuerpos intermedios establecida en el art. $1^{\circ}$ de la Constitución. 
Por tanto, es posible, desde el punto de vista constitucional, que exista alguna forma de control sobre la calidad y eficacia con que las diversas instituciones de educación superior cumplen sus tareas, colocando ciertos límites al ejercicio de la autonomía de estas entidades, en la forma y oportunidad que determine el legislador ${ }^{2}$.

$\mathrm{Al}$ aplicarse esta garantía, Los padres tienen el derecho de escoger el establecimiento de enseñanza para sus hijos (huelga decir que los alumnos mayores de edad son libres de elegir por sí mismos el establecimiento donde desean desarrollar estudios superiores). Para ejercer debidamente este derecho, los potenciales estudiantes requieren contar con información oficial acerca de las instituciones y carreras que, por haber cumplido con los requisitos legales, cuentan con reconocimiento oficial, más allá de la publicidad que realizan las instituciones de educación superior.

El ministerio de Educación es el órgano público encargado de entregar esa información oficial que las familias usuarias del sistema de educación superior requieren para el ejercicio del derecho comentado ${ }^{3}$, en virtud de sus facultades legales de otorgar el

2 Ver Actas de la Comisión de Estudios para la Nueva Constitución (C.E.N.C.), Sesión No 148, Guzmán, J.: "Hay dos alternativas: una, hacer Fe en la Autonomía Universitaria y pensar que mientras el Estado mantenga el reconocimiento oficial de la universidad, se presume que esa universidad hace buen uso de su autonomía en los campos académico, administrativo y económico, y que el Estado no tiene facultad para intervenir o para imponer nada al interior de esas decisiones universitarias. La segunda alternativa es que exista alguna forma de control con imperio sobre las universidades, tendiente a garantizar dos grandes objetivos: primero, la seriedad del nivel superior de la enseñanza y segundo, el aprovechamiento de los recursos económicos que están envueltos en la vida universitaria". Guzmán, desde el punto de vista doctrinario, siente atracción por la primera fórmula, la autonomía plena, en la que si una universidad hace tan mal uso de ella, el Estado puede privarle de su reconocimiento oficial por el mismo instrumento jurídico a través del cual se le concedió. Sin embargo, le parece que es tan complejo tomar una resolución de esta índole que, al mismo tiempo, pudiera ser tan injusta al abarcar a toda la universidad cuando en realidad el foco del problema puede estar radicado en un rubro específico, que cree que esta fórmula no sería operante desde el punto de vista práctico, y en los hechos llevaría a que no haya medio alguno de control sobre la idoneidad de los estudios universitarios o el buen aprovechamiento de los recursos. Si se opta por un Sistema de Coordinación, en la práctica se va a obligar a las universidades a entrar en un diálogo entre ellas, de lo cual sólo podrá salir un resultado en la medida en que haya acuerdo: "ése es el carácter del Consejo de Rectores actual, un Consejo de Coordinación”. Sugiere un organismo autónomo, con imperio, en que uno de los límites más eficaces estaría garantizado por una integración mayoritaria de representantes de la vida universitaria chilena y de organismos afines, como son los colegios profesionales y las organizaciones científicas.

3 Ver Ley No 18.956, orgánica del ministerio de Educación. 
reconocimiento oficial a las instituciones y la de fiscalizar el cumplimiento de sus fines.

Para terminar este acápite relativo a las normas constitucionales, conviene mencionar el derecho a la educación, que es un derecho público subjetivo, un interés propio de la voluntad de las personas, jurídicamente reconocido y protegido por el Estado, en cuanto condición necesaria para la realización de los proyectos de vida individuales. La CP, en su artículo 19, número 10, inciso quinto, señala:

a) "Corresponderá al Estado, asimismo, fomentar el desarrollo de la educación en todos sus niveles; estimular la investigación científica y tecnológica, la creación artística y la protección e incremento del patrimonio cultural de la Nación"; y

b) "Es deber de la comunidad contribuir al desarrollo y perfeccionamiento de la educación”.

\section{LA LOCE: EL MARCO LEGAL DEL POSGRADO EN CHILE.}

La Ley 18.962, Orgánica Constitucional de Enseñanza (LOCE), que fija -de acuerdo al mandato constitucional- los requisitos para el reconocimiento oficial de las instituciones educacionales de todos los niveles, establece algunas regulaciones básicas para los estudios de posgrado, dejando la mayor parte de los contenidos, definiciones y procedimientos a la libertad de enseñanza y autonomía de las instituciones.

Enseguida se detallan las normas que, directa o indirectamente, regulan la oferta de programas de posgrado: De partida, el artículo $4^{\circ}$ define la enseñanza formal como "aquélla estructurada científicamente, que se entrega de manera sistemática". Sus niveles "aseguran la unidad del proceso educativo y facilitan la continuidad del mismo durante la vida de las personas”. A su turno, el artículo 50 establece que la enseñanza formal se denomina regular "cuando sus niveles se imparten a educandos que cumplen con los requisitos establecidos, de ingreso y de progreso en ella". De lo anterior se desprende que la formación de posgrado corresponde a la enseñanza formal y regular. 
Conviene recordar, asimismo, que la legislación relativa al sistema de educación superior en Chile, dictada a partir de 1980 (DFL 1/80, Ed.), depositó de manera exclusiva y excluyente en las universidades reconocidas por el Estado la habilitación profesional en determinadas carreras que exigen licenciatura previa, así como el otorgamiento de posgrados $^{4}$.

Por su parte, el artículo $31^{\circ}$, inciso cuarto de la LOCE, señala que las universidades pueden otorgar "toda clase de grados académicos, en especial, de licenciado, magister y doctor" y define dichos grados:

a) "El grado de magister es el que se otorga al alumno de una Universidad que ha aprobado un programa de estudios de profundización en una o más de las disciplinas de que se trate. Para optar al grado de magister se requiere tener grado de licenciado o un título profesional cuyo nivel y contenido de estudios sean equivalentes a los necesarios para obtener el grado de licenciado"; y

b) "El grado de doctor es el máximo que puede otorgar una universidad. Se confiere al alumno que ha obtenido un grado de licenciado o magister en la respectiva disciplina y que hay a aprobado un programa superior de estudios y de investigación; y acredita que quien lo posee tiene capacidad y conocimientos necesarios para efectuar investigaciones originales. En todo caso, además de la aprobación de cursos u otras actividades similares, un programa de doctorado deberá contemplar necesariamente la elaboración, defensa y aprobación de una tesis, consistente en una investigación original, desarrollada en forma autónoma y que signifique una contribución a la disciplina de que se trate".

De este modo, la ley fija las competencias de los diferentes tipos de instituciones de educación superior, a la vez que establece los requisitos para la validez de los grados académicos otorgados por aquéllos. Dos importantes consecuencias se derivan de estas normas: primero, sólo las universidades legalmente constituidas en Chile pueden otorgar grados académicos de licenciado, máster y doctor; y segundo, dichos grados no son los únicos que pueden otorgar las universidades.

4 Adicionalmente, el DL No 3631, publicado en el Diario Oficial el 28 de febrero de 1981, establece que ninguna entidad, institución o establecimiento podrá denominarse universidad o emplear en los títulos que otorgue y en el desarrollo de sus actividades la expresión universitario, si previamente no se ha constituido como universidad en conformidad a la ley. 
El proceso de reconocimiento oficial, a través del cual el Estado regula la entrada de nuevas entidades al sistema, se complementa con mecanismos de examinación -por parte de universidades tradicionales- prevista en el DFL No 1 de Educación de 1980; o de acreditación -por parte del Consejo Superior de Educación-según la LOCE. Las nuevas universidades deben superar un proceso de acreditación, previsto en el párrafo $2^{\circ}$ del Título III (arts. 32 y siguientes de la LOCE), proceso que comprende la aprobación del proyecto institucional y la evaluación periódica de su avance y concreción, con miras a la obtención de la plena autonomía.

Según la ley, la acreditación cubre las variables más significativas del desarrollo de cada proyecto institucional: infraestructura, equipamiento, recursos económicos y financieros, recursos para la docencia (bibliotecas, laboratorios, talleres, equipos computacionales), cuerpo académico, servicios, etc., en todo lo referido a las funciones de docencia, investigación y extensión. Así, los programas de posgrado son objeto de una supervisión integral, en la medida que se creen durante la primera etapa de vida institucional. El proceso de acreditación dura seis años, plazo a cuyo término si la evaluación es satisfactoria se otorgará la autonomía, o, en caso contrario, se podrá extender este proceso hasta por un máximo de cinco años más.

Por último, en lo tocante a la LOCE, de acuerdo con el artículo 75 de la misma, corresponde a las instituciones autónomas de educación superior impartir independientemente programas de estudio conducentes a los grados referidos, y velar por sí mismas respecto del cumplimiento de las regulaciones legales indicadas.

En lo relativo a especializaciones, postítulos, diplomados y otros grados académicos, como la ley nada dice y las actividades académicas de la universidad están cauteladas por la garantía constitucional de Libertad de Enseñanza, rige plenamente el principio de la autonomía y libertad académica, sin otras limitaciones que las impuestas por la moral, las buenas costumbres, el orden público y la seguridad nacional. 


\section{LA ACREDITACIÓN DE POSGRADOS DE UNIVERSIDADES AUTÓNOMAS:}

El ministerio de Educación creó, por Decreto No 225 de 1999, la "Comisión Asesora en materia de Evaluación de la Calidad de Programas de Posgrado de universidades autónomas" (CONAP) y, mediante Resolución Exenta No. 3.380, de 2000, fijó las "Bases, criterios y procedimientos para la evaluación experimental de la calidad de los programas de posgrado de las universidades autónomas".

Se fundamenta la creación de esta comisión en la LOCE, en cuanto ella fija requisitos para el reconocimiento oficial de instituciones de educación superior, define los grados que las universidades pueden otorgar; y en la ley $\mathrm{N}^{\circ} 18.956$, según la cual el ministerio de Educación debe velar por el cumplimiento de las normas legales y reglamentarias que regulan la educación superior, en el ámbito de su competencia, y otorgar el reconocimiento oficial a las instituciones de este nivel. El diagnóstico que le sirve de base es el aumento de las certificaciones que se ofrecen y la ausencia de un sistema de evaluación que promueva la calidad de los programas en universidades autónomas. Por último, se explicita que estas acciones se enmarcan en el Componente de Aseguramiento de la Calidad, del Programa Mejoramiento de la Calidad y la Equidad de la Educación Superior (MECESUP), que lleva a cabo el ministerio de Educación, con un crédito del Banco Mundial.

El artículo $1^{\circ}$ de este decreto enfatiza el carácter asesor de dicha comisión. Cabe recordar que una abundante jurisprudencia de la Contraloría ha señalado que estas comisiones no pueden cumplir funciones de carácter resolutivo ni permanente. Los objetivos que ella debe cumplir son básicamente dos:

a) Proponer al ministro de Educación las bases institucionales y el diseño de un proceso de evaluación académica de los programas de Maestría y Doctorado que impartan las instituciones autónomas; y

b) La puesta en marcha de ensayos metodológicos y experimentales, para tal efecto.

Respecto a los ensayos metodológicos y experimentales, las instituciones autónomas de educación superior podrán participar 
voluntariamente. No obstante, para los efectos de asignación de las Becas CONICYT de posgrado en Chile, se ha hecho obligatoria la participación en estos ensayos metodológicos, lo cual nos parece razonable desde el punto de vista de una adecuada asignación de recursos públicos (bastante escasos, por lo demás).

Finalmente, la comisión se creó, en principio, por dos años, que fueron prorrogados a cuatro, contados desde su creación, por decreto supremo N $^{\circ} 541$ del 20 de septiembre de 2000, de Educación. Sin embargo, el proyecto de ley sobre establecimiento de un sistema nacional de aseguramiento de calidad, que actualmente se tramita en el Parlamento, propone radicar la acreditación de los programas de posgrado en una agencia pública única, adscrita en el Consejo Superior de Educación y que acreditaría tanto programas de pregrado como de posgrado distinta de la comisión especializada que actualmente ha estado sirviendo esta función bajo el amparo institucional de CONICYT.

\section{A MODO DE CONCLUSIÓN}

En otra parte hemos dicho que, desde el punto de vista de las políticas públicas -y reglas constitucionales- corresponde al Estado la producción de un conjunto de bienes públicos. Respecto de las instituciones de educación superior y sus programas, compete al Estado proveer -al conjunto de la sociedad, al mercado y a los consumidoresde información fidedigna acerca de sus objetivos y la forma en que se están cumpliendo, en referencia, por ejemplo, al proceso y los recursos necesarios para su logro ${ }^{5}$.

En el caso del posgrado, el sistema legal vigente delega la producción de ese bien en el mercado. Se espera que el sistema de educación de cuarto nivel provea esa información de manera espontánea y transparente, o bien, desde otra perspectiva, se obliga a los consumidores a obtenerla por sí mismos. La información pública disponible se encuentra bajo la fórmula del reconocimiento oficial prevista en el texto constitucional y se complementa con la

5 Ver Peña, C. y León, J, "Regulación de las profesiones: ¿Estado o Mercado?", Revista CSE en No 16, Santiago, Chile, Julio 2002. pp. 51-63 
segmentación que se produce gracias a la obtención de la plena autonomía, por parte de algunas instituciones más consolidadas, previo cumplimiento de los requisitos de acreditación o examinación previstos en la LOCE.

A mi modo de ver, no hay mayor motivo para desconfiar de lo que han estado haciendo las universidades en este ámbito: en efecto, aunque operan con un alto grado de autonomía y desregulación, ellas han actuado hasta aquí y en materia de posgrados con seriedad. Por esta razón, el número de nuestros estudiantes de posgrado, y, en especial de doctores, es bajo y debería tender a aumentar.

Por otra parte, el sistema de acreditación de posgrado, con perfil académico y para los efectos de asignación de becas, que ha estado funcionando en CONICYT ha sido eficiente respecto a los fines para los que fue creado.

En rigor, el diseño correcto de un sistema de educación superior debería seguir una forma piramidal, desde el punto de vista de la regulación y también del volumen de financiamiento. Fuerte y amplia en la base (educación primaria y secundaria), con el fin de asegurar el cumplimiento de los objetivos fundamentales y contenidos mínimos, dar coherencia al sistema educacional y resolver el problema de la equidad, mejorando las oportunidades para todos; y con presencia decreciente hasta llegar al nivel superior -la formación de maestrías y doctorado-donde las necesidades de regulación y financiamiento son menores, en cuanto estamos en presencia de interesados con mayor información para elegir y discriminar el servicio educacional que quieren contratar y con capacidad relativa para financiar directamente $\mathrm{u}$ obtener ayudas para financiar sus estudios.

En este nivel, el financiamiento y la acreditación deberían mantenerse vinculados en CONICYT, como lo están hoy, para promover programas y formación de cuadros académicos de alto nivel en todas las disciplinas.

En tal sentido, no nos parece adecuada la propuesta legislativa que busca radicar la acreditación de posgrados en una agencia estatal única, cuyo foco principal será la acreditación de carreras de pregrado, 
con la consiguiente pérdida que se producirá de la relevante experiencia y especialización desarrollada por la CONAP, amén de la confianza que ésta ofrece hoy a todos los actores del sistema.

Solo con un marco institucional apropiado, el posgrado podrá constituirse en el eslabón que permita a las universidades hacer converger las actividades de docencia de pregrado con la investigación; promover publicaciones; potenciar la comunidad académica; generar nuevos lazos con los graduados y titulados; recoger las nuevas tendencias mundiales y traspasarlas al quehacer académico, entre otros beneficios y aportes al desarrollo nacional. 
